\title{
An option for diagnosis in non-palpable lesions of the breast
}

\author{
Allende S*, Pérez JTS, Sánchez PEB, Gutiérrez SH, Mazorra AA and Moreno DM \\ Hospital clínico quirúrgico, la habana, Cuba
}

\begin{abstract}
In the face of a non-palpable mammary lesion requiring a diagnostic biopsy, the ideal guiding method should be assessed to access it. Currently, several methods are used: stereotaxy, ultrasound, MRI, among others. The next step is to choose the most appropriate biopsy technique. Fine needle aspiration biopsy, coarse needle biopsy, vacuum assisted biopsy, etc. The most classic and reliable technique is the surgical biopsy with previous marking, which can be done with harpoon, marking with radioactive substances or stereotaxy.

This work, it considers a technique of marking by planimetry of the lesion, where the radiologist orients the surgeon on the site where the lesion is three-dimensional and posterior to the exeresis of the same one carries out study mammographic to the surgical piece, to corroborate the presence of the same and in turn mark it with a needle so that the pathologist performs the pertinent cuts of the tissue, being of great use in the microcalcifications, with low cost because it is done by ambulatory surgery propitiating the Incorporation of the patient quickly to its usual work.
\end{abstract}

\section{Introduction}

Between malignant tumors more frequently and with greater impact day is today to breast cancer, which in addition to a fearsome disease, is at present, an event with high economic and social impact $[1,2]$.

Breast cancer is a multifactorial, polymorphous and heterogenous disease that develops and grows in a field of individualized, specific biological expressions and well-defined evolutionary phases that make up a wide variability in the history natural of this neoplasia, by what some claim that it is a systemic disease of home, with its first clinical manifestation in the breast [3]. It is the most frequently diagnosed malignancy and important cause of morbidity and mortality in women.

In the United States, breast cancer is the most frequently diagnosed malignancy and the second leading cause of death by cancer [2].

It is estimated that approximately 183,000 women are diagnosed with invasive breast cancer each year, and of these, approximately 41,000 will die from the disease $[1,2,4]$.

In Cuba there is a significant risk of getting sick and dying from breast cancer. According to data offered by the National Cancer Registry, the incidence rate was 38.0 per 100.000 women and the mortality of 17.2 by 100.000 women in 2017 [5].

In view of the heterogeneity it presents both in terms of its biological profile and its clinical progression, it is necessary to have parameters that collaborate in the elaboration of a certain prognosis and an effective treatment.

Despite the multiple efforts in the field of research carried out in all the latitudes of the planet, the early diagnosis remains the cornerstone for improving the disease-free life and interval in women with this ailment $[3,4]$.
Precisely the contemporary imaging has marked a milestone in the early diagnosis of the disease, because with the development of preventive programs, (Applied with the peculiarities of each country) aimed at diagnosis in early stages, mainly in non-palpable lesions, or in the subclinical phase of the disease [6-8].

\section{Development}

The diagnosis by the image of mammary lesions has changed in recent years, conventional mammography has benefited from digital technology, ultrasound have substantially improved their quality and MRI has been introduced into the Diagnostic algorithms [7,8].

Each one of these techniques has its individuality for the diagnosis of the conditions of the mammary gland, but without doubt in the screening, it is the mammography, who occupies the Cimero place for diagnosis of the subclinical lesions.

The extensive use of mammography, not only as a complementary means in diagnosing palpable lesions of the mammary gland, but also as a screening procedure for breast cancer, has significantly increased the detection of injuries Clinically not palpable mammary, achieving a considerable increase in definitions in stages 0 and I (carcinomas in situ and atypical hyperplasia among others) [8,9].

Mammography evidences four imaging signs that put-on alert about the possible presence of the disease, which are:

${ }^{\star}$ Correspondence to: Allende $\mathrm{S}$, hospital clínico quirúrgico, la habana Cuba, E-mail: teresasp@infomed.sld.cu

Key words: planimetry, breast cancer, non-palpable lesions

Received: February 01, 2019; Accepted: February 11, 2019; Published: February 14,2019 
- Presence of tumor or nodule.

- Presence of Microcalcifications.

- Asymmetry of the mammary pattern.

- Distortion of the mammary pattern or architectural distortion.

Two images of the form are exposed, more common in that is discovered the disease, from the point of view Imaging (Figures 1 and 2). At the onset of any of these signs, then imposes the realization of biopsy for a conclusive diagnosis. There are several methods to obtain the sample of tissue, which would definitively with the histological diagnosis of the patient in question $[9,10]$.

The establishment of the BI-RADS categories of the American College of Radiology since the year 1990, has been an important

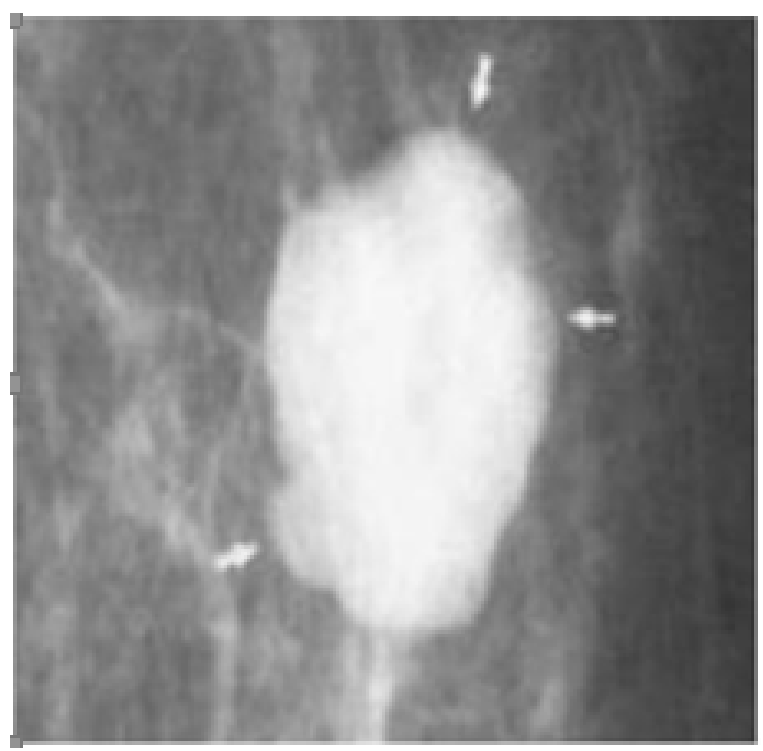

Figure 1. Presence of Tumor or nodule

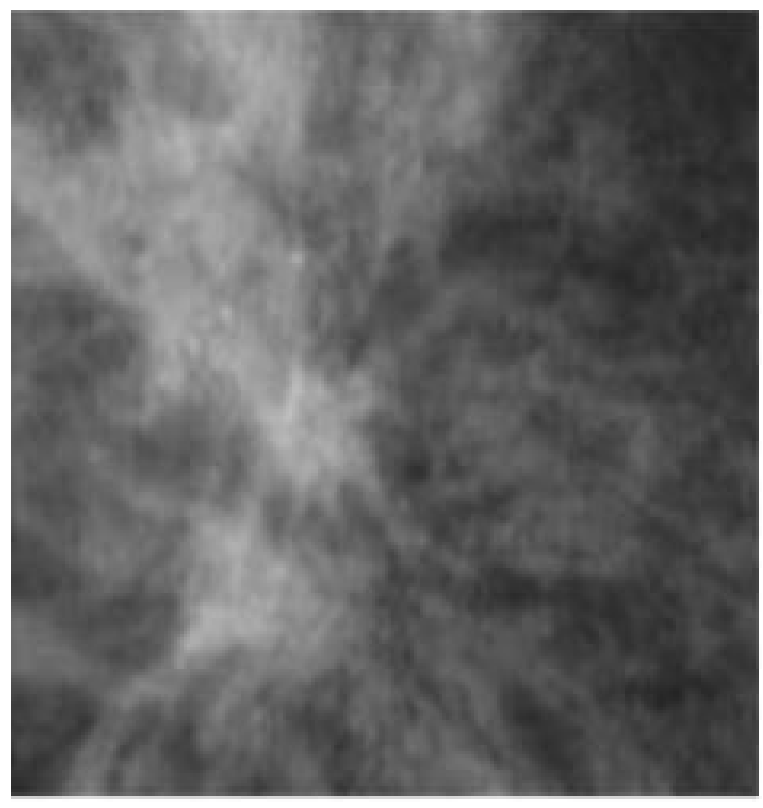

Figure 2. Presence of macrocalcifications milestone in the classification of breast lesions, by allowing to establish guidelines for their diagnosis. Category 1 corresponds to the normal breast, without injury; Category 2 to benign inconsequential injuries; Category 3 to likely benign lesions with positive predictive value (VPP) for cancer less than 2\%; Category 4 to suspicious lesions with a variable VPP between 2 and $85 \%$, and finally category 5 , highly suspected of malignancy, with a VPP higher than $85 \%$. Obviously, there is no point in performing a biopsy of the lesions classified in categories 1 or 2 . However, it is necessary to perform biopsy of category 4 or 5 lesions given their moderate or high VPP for cancer. The management of Category 3 lesions is more controversial, since although the first option may be short-range monitoring (every six months for two years), there is an alternative to practicing a puncture biopsy that avoids the anxiety of controls Mammographic [9].

Non-palpable lesions are those that represent a challenge in obtaining sample for histological examination.

This is how different guidance systems are proposed for obtaining the sample, as mentioned below:

- Through mammography.

- Using Stereotaxy.

- By Ultrasound.

- Use of harpoons.

- Iodine seeds 125

- Through surgery

All the methods indicated have their advantages and disadvantages, they vary in their costs and they are adaptable in the conditions of the place where they are carried out [10].

In this work it is intended to expose the planimetry as a possible alternative, in the surgical biopsy, to lack of other possibilities.

This technique is to perform the excision of the lesion reported by the radiologist, through surgery, which places the surgeon threedimensional the site of the lesion, and subsequently to the excision of this, perform a mammographic study of the surgical piece, to corroborate the presence of the same, comparing with the study mammographic previously executed; Confirmed the presence of the previous radiological sign, which suggested the study of the lesion, the same is marked with a needle and is sent to the Department of Pathology for its histological diagnosis.

This method has resulted, low cost among others and implies an option to take into account in places where there is no high resources, also is done by ambulatory surgery achieving the immediate incorporation of the sick to their usual activities.

To the Mammographic study, where the subclinical lesion was detected, the pertinent measurements are made, which orient the surgeon, about the location of the lesion tridimensionally, as shown in Figures $3 \mathrm{~A}$ and $3 \mathrm{~B}$.

After the exeresis completed, another image study is made to the extracted piece to check the presence of the irregularity that caused the procedure and is marked with a needle to specifically orient the pathologist where the abnormality is Figures $4 \mathrm{~A}$ and $4 \mathrm{~B}$. 


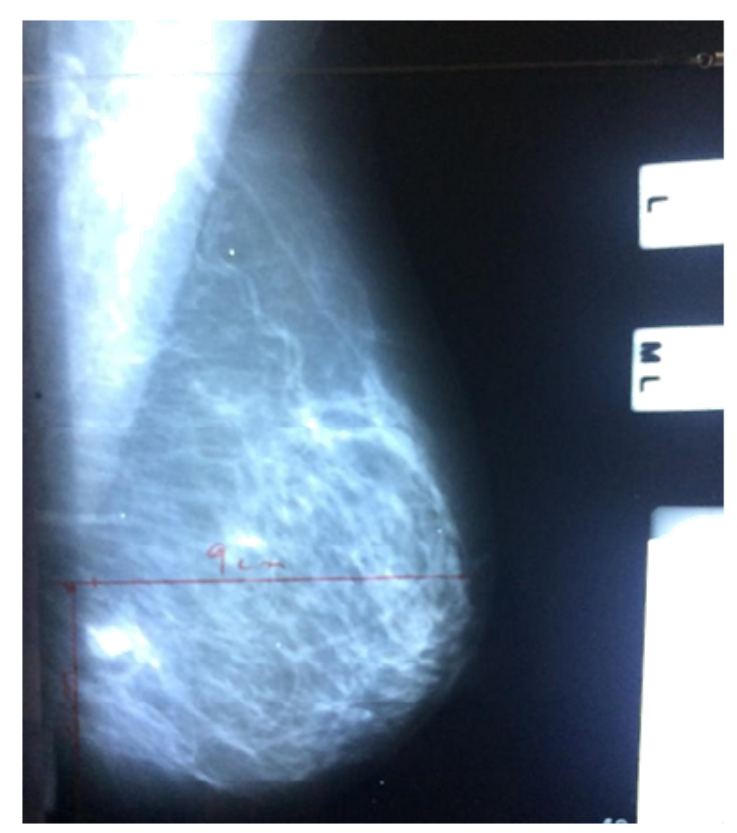

Figure 3A. Side view, where the distance of the nipple is marked to the lesion. $9 \mathrm{~cm}$

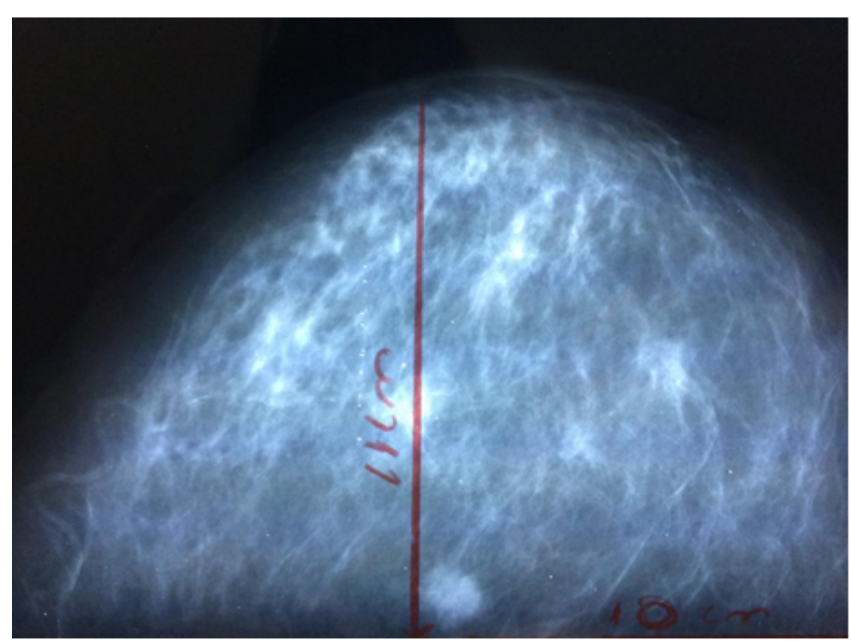

Figure 3B. Skull-caudal view

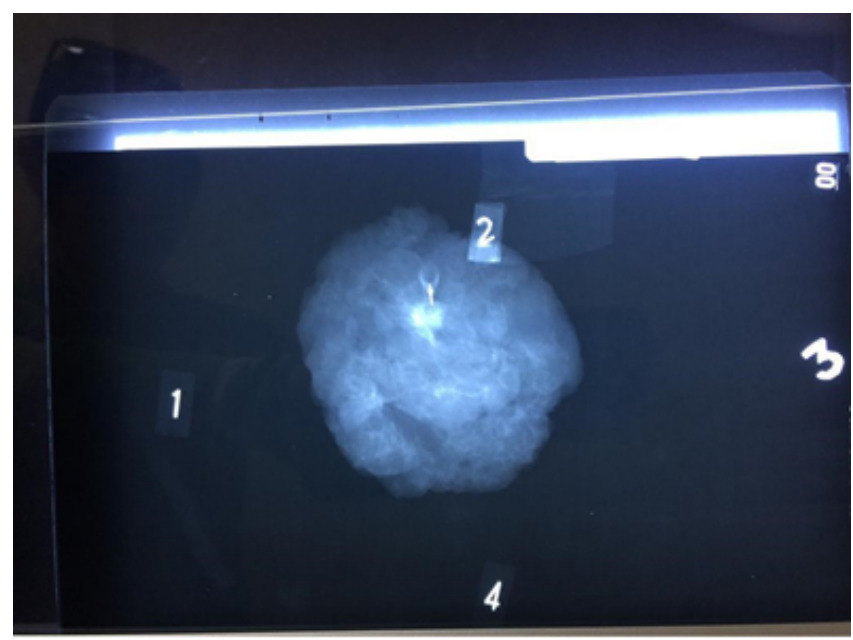

Figure 4A. Needle lesion marking

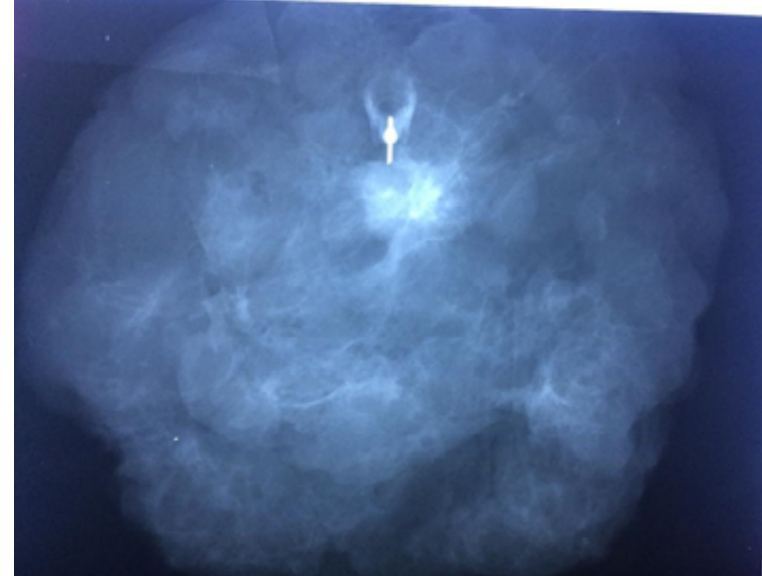

Figure 4B. Needle lesion marking, magnified

\section{Conclusion}

In the face of a non-palpable lesion of the mammary gland, which needs biopsy, it is necessary to plan what is the most susceptible guiding method (Stereotaxy, ultrasound, MRI, marking with radioactive substances, among others) and in second place It could be the technique that provides the best results in each case specifically. It is difficult to establish a defined protocol, as it could result in irreparable errors due to the different lesions that occur, (nodules, microcalcifications, etc). In this work only intends to incorporate another method capable of obtaining a sample of tissue as ideal as possible to reach the histological diagnosis that is ultimately the golden Rule, which guidelines the final conduct for those who are sick who have a diagnosis breast cancer.

In short, it must be the individual experience of each center that rule the protocol of diagnostic management of these injuries.

\section{References}

1. World Health Organization (2015) Cancer prevention and Control. Provisional Agenda Item 13. 12, 58th World Health Assembly, Geneva, Switzerland.

2. Leis HP (2014) Epidemiology of Breast cancer. New York Times Medical publishes, 3-10.

3. MedlinePlus [Internet page]. "Breast cancer". Medical Encyclopedia in Spanish.

4. National Cancer Institute, General Information on Breast cancer (breast).

5. Ministry of Public Health: national Registry of Cancer (2017) Department of Statistics. MINSAP. Havana.

6. Ramos JM, Guerrero FS, Rodríguez NR, Isaza EI (2003) Biopsy of non-palpable mammary lesions by means of a harpoon mark guided by Stereotaxy. Annals Radiol México 1: 23-27.

7. Vega Bolívar Interventional diagnosis in Breast pathology. Published by Elsevier España SL.

8. Aznar JMP, Zamora BO, Chiva GE, García NL (1995) Pre-surgical marking of clinically occult mammary lesions: review and Results REV. SENOLOGIA and Paton. MAM 8: 1.

9. American College of Radiology (2006) BI-RADS $($. Breast image data reporting and recording system. Reston VA: ACR; 2003. (Ed. esp.) Madrid: SERAM.

10. Bolívar AV, Alonso-Bartolomé P, García EO, Ayensa FG (2005) Ultrasound-guided core needle biopsy of non-palpable breast lesions: A Prospective analysis in 204 cases. Acta Radiol 46: 690-5. [Crossref]

Copyright: (C2019 Allende S. This is an open-access article distributed under the terms of the Creative Commons Attribution License, which permits unrestricted use, distribution, and reproduction in any medium, provided the original author and source are credited. 